\title{
International Lattice Data Grid: Turn on, plug in,and download
}

\section{C.M. Maynard*}

EPCC, School of Physics, University of Edinburgh, UK

E-mail: c.maynard@ed.ac.uk

In the beginning there was the internet, then came the world wide web, and now there is the grid. In the future perhaps there will be the cloud. In the age of persistent, pervasive, and pandemic networks I review how the lattice QCD community embraced the open source paradigm for both code and data whilst adopting the emerging grid technologies, and why having your data persistently accessible via standardized protocols and services might be a good idea.

The XXVII International Symposium on Lattice Field Theory - LAT2009

July 26-31 2009

Peking University, Beijing, China

*Speaker. 


\section{A brief history of everything}

The International Lattice Data Grid (ILDG) is a project which has been running for seven years. The basic idea is to use technology such as the grid to enable different groups to share their data. It is a volunteer effort, in that there is no central authority, nor is there any central funding for effort. There is no central data repository. Each participating group and individual contributes what they can in terms of effort, services and data, to make an aggregation of data accessed by defined services which makes up the ILDG. In this paper I review the key ideas and explain the rationale behind the project and motivate why this activity is useful, in addition to our more usual activities of writing computer code and science papers. Indeed, a more systematic approach to data management is a necessary addition! A technical paper on the ILDG services and protocols is available on the e-print archive [1].

In the beginning was the Internet. It was and is a way of connecting machines together, with multiple routes to different machines. The basic ideas were developed in the late sixties and early seventies, and by the nineties a global Internet existed, which continues to grow to this day. Then came the world wide web, which utilised hyper-text markup language as a way of sharing information. The world's first web server was at CERN and went online in 1991. In the late nineties the "information super-highway" began to be talked about. In academic circles, this became known as the grid. In the business world this became known as web-services. In a direct analogy with the world wide web, these services and protocols are for sharing data and services which may act on data. The future, potential next stage of evolution is sometimes called cloud computing. Scalable, transparent, virtual services are provided to users, who access there data via these services from mobile devices, phones, laptops etc. Everything lives in the cloud and is operated on by clients.

These networks are persistent, pervasive and pandemic. In an ideal world so would be our data. Our data access patterns and methods should reflect this. How do we access our data? The same way we did a decade or more ago ${ }^{1}$, with ssl terminal clients (ssh) and copy protocol (scp). However, our data has changed. We are facing a data explosion. Data volumes have certainly increased as the size of the lattice has increased, we now routinely deal in Tera Bytes, and numbers of configurations belonging to an ensemble have also increased. We also have to cope with increased data complexity. We have many, many different ensembles with different quark masses, gauge couplings and lattice volumes, with many, many different measurements on each ensemble. Moreover, the ever increasing cost of data generation and the complexity of the analysis forces groups to join together to share resources in terms of both computer and man power. These "Megacollaborations" can be distributed across continents and time-zones, as can the data. Some tools to help manage access to the data are becoming a necessity.

How did this activity get stared? The twin philosophies of open source code and open data started to gain credence around the turn of the century. The open source code movement was well known to the lattice community. Many people are familiar with the Linux operating system, and the GNU compilers, as well as many of the everyday tools such as open ssl. Would any of the open source ideas be relevant to actual physics code? In terms of computation, all the community does the same thing, viz. parallel linear solvers. There is only a relatively limited amount of technical effort available, most of the community is interested in doing physics rather

\footnotetext{
${ }^{1}$ Technologically equivalent with the stone age
} 
than assembler coding per se, so it does make sense to avoid duplicating work. Many physics codes are now open source. For example, consider the USQCD [2] common programming environment. Development of architecture optimised kernels are centrally resourced and made available to the community via standardised interfaces such that legacy codes, the CPS, MILC, and Chroma, can all call these kernels. The codes are legacy in the sense of existing before the common programming environment. They represent many thousands of "man-hours" of physics coding, which is now protected by the development of these kernels.

Open data is a relatively new concept. Many groups had shared data before, but the MILC collaboration stated to make their data freely available via the gauge connection [3]. This was ground breaking, in that anyone could use their gauge configurations. Many people have benefited from their approach and giving away their data has not harmed MILC's scientific program. Indeed, they have gained such kudos that other groups have started to copy this strategy.

The emerging grid technologies, such as globus [4], made distributed computing and in particular distributed data systems a possibility. Richard Kenway made a proposal that the community should get together and harness these ideas to make sharing data technically feasible. At an open meeting at the lattice conference in Boston in 2002 [5] this was favourably received and the International Lattice Data Grid was born!

\section{What is the ILDG?}

There is an organisation, and an aggregated data repository called the ILDG. Let us consider the organisation. Each group taking part in the ILDG decides what data is available to others. As such, the ILDG has no formal role. Groups collaborate informally, based around two working groups. One for the metadata and one for the middleware. Individual groups were already starting to build their own, or use existing, data grid infrastructures. The Adelaide group was developing a web-portal. Several German groups had combined to make LATFOR, which had its grid arm, LDG. The Japanese groups had started the Japanese Lattice Data archive, which became the Japanese lattice data grid. UKQCD was developing its own grid system, QCDgrid, later DiGS. The US groups had formed USQCD which had its own data repository functionality. The middleware for each of these systems was dictated by national funding considerations. The ILDG is an aggregation of these middleware systems, and making them interoperable is the key task for the ILDG. The ILDG data repository can be thought of as a 'grid-of-grids'.

There are three required conditions for the ILDG to exist. Firstly trust. This is already established in the community. We already know each other, and review each others work and grant proposals. We may not always agree on the science, but it is almost inconceivable that some would claim gauge configurations another group had generated as their own. The second condition is altruism. There needs to exist the political will to make the data available. This is perhaps not so hard, as we already share code and publish our results and ideas freely. However, there is effort required collectively to build the infrastructure and individually to publish, or make available, the data. The third condition is reward. How to reward those making the data available, both individually and as a collaboration. Typically this is done by users of the data citing a designated paper. This is the mechanism used to reward those who are involved with machine building or coding effort and the result should be a highly cited paper. 


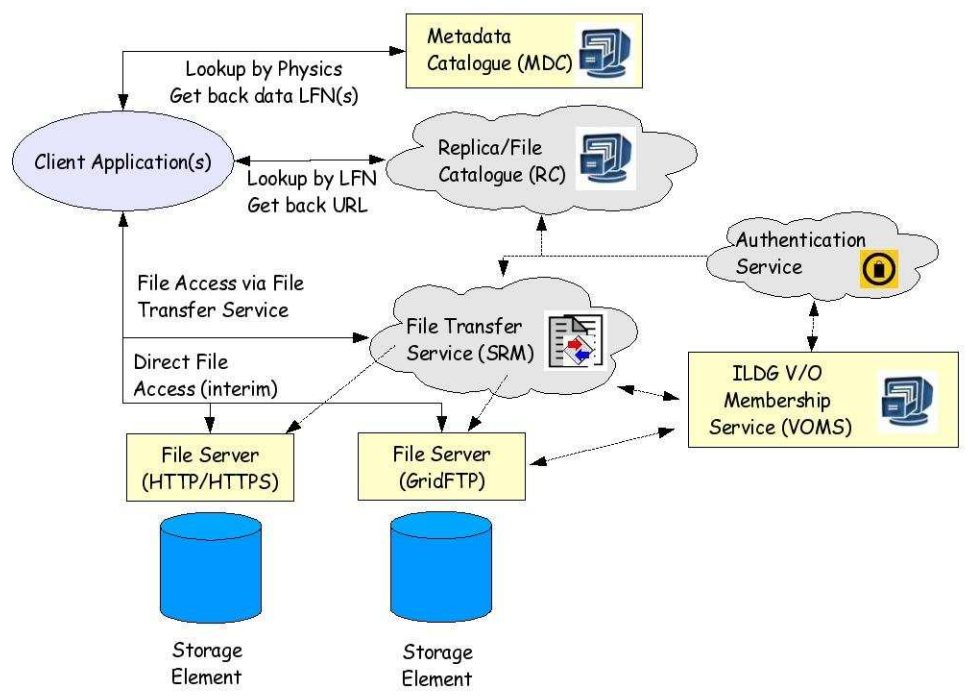

Figure 1: A schematic diagram showing the architecture of the ILDG services

There are three key ideas or concepts to make this work. Firstly a standard format for the data. It really doesn't matter what this format is as long as everyone can read and write data in this format. One of the first activities of the metadata group was to agree this format. For configurations this is the SciDAC LIME records, where the gauge configuration data itself is the NERSC data layout, storing the full $3 \times 3 S U(3)$ matrix. Secondly, there must be a standard description of the metadata. This is a semantic description of the data, which can be processed by an application. A more detailed discussion of the metadata will follow. Thirdly, standard interfaces to services are required, so that the same query can be submitted to all the metadata catalogue services (MDC) and File Catalogue Web services (FC), what ever underlying middleware system is running them, and critically, supply an response in an standard format.

A complete a complete description of the architecture can be found on the ILDG web-pages [6], is reviewed in [1] and is shown in figure 1. Access to ILDG services is granted to users who belong to the ILDG Virtual organisation (VO). To join, a user needs an X509 certificate from their regional/national academic certification authority. The distinguished name (DN) which is part of the certificate and is the user's identity on the grid is registered with the ILDG VO, and confirmed by a representative from the regional grid to which the user belongs. More details on how to do this can be found on the ILDG web pages [7]. Who is a member of the ILDG is thus controlled by the regional grid organisations.

To access data, a user starts the client application which can submit physics queries to the MDC. This returns the Logical File Name (LFN) of the data, this a unique identifyer for a particular datum. The MDCs are public, and can in principle be read by anyone, but many actually require authentication, i.e. who is reading the MDC, even if the information is public. This is controlled via the authentication service, which talks to the ILDG Virtual organisation using VOMS. The client application then submits the LFN to the FC which returns a URL which encodes the location of 
the data. A file transfer is initiated between the storage location chosen by the FC and the location chosen by the client.

\section{Metadata}

The key concept for the ILDG is metadata. Metadata literally means data about data. This is crucial when sharing and archiving the data. What data is contained in the file? How was it created? What was the physics, the algorithm, computer code and machine? Who created the data? and when? These are all obvious questions about the data. When using data created by another group it is important that this information is recorded in a standard way, so that it can be understood. To motivate the need for a standard further, let us consider the extreme case of no metadata. All the files for the configurations from many ensembles have random strings for names, and all live in the same directory. This is clearly useless. The obvious thing to do is to organise the files into different directories for each ensemble, and encode some of the metadata into the names of the directories and files. The files code have the position in the Markov chain denoted by the trajectory number. This is constructing a basic scheme or set of rules for describing the data.

This basic and commonly used scheme has "meaningful" file names, i.e. the metadata is encoded in the file names. The directory structure can be used to encode some structure or hierarchy into the scheme, but it is not completely flexible. The directory structure is a tree, which doesn't map terribly well onto the hierarchies inherent in lattice QCD data. For example, if there were ensembles with multiple volumes and quark masses, should the directory structure be volume/mass or vice versa? What happens if data with another flavour of quark is added? It doesn't fit into the structure, because the scheme is not extensible, i.e. new data cannot be added without changing the scheme in such a way that the existing metadata has to be modified.

D52C202K3500U010010_LL3450X_FL3400X_CMesonT00T31 is a UKQCD file name for a meson correlation function. It is an example using the file names to encode the metadata, and is a broken scheme. What does $\mathrm{X}$ denote? Originally this position in the file name was used to describe the action. $\{W, R, C\}$ meant Wilson, Rotated or (tree-level) Clover. However, there then turned out to be many sorts of Clover action, depending on the value of the coefficient. So this was denoted with X meaning "none of the above". The scheme is broken, there is no where to record the value of another coefficient. To do so consistently would require a new scheme, and old meson file names would not fit in this new scheme. Later, UKQCD started doing dynamical fermion simulations, so some metadata for the quark action was recorded in the first part of the name, D denotes dynamical. $\mathrm{C} 202$ denotes $c_{S W}=2.02$, but only to three significant figures. The actual value in the simulation was $c_{S W}=2.0171$. A simple scheme based on meaningful file names is a sensible first start, but is ultimately inadequate as there is a poor match between the hierarchical nature of lattice QCD data and the tree structure of a directory scheme and critically, the lack of extensibility of such a scheme.

What is required from a metadata scheme? There should be sufficient information that the data can be discovered, accessed or found from the metadata, i.e. where is this data. The nature of the data should be encoded in the metadata. This is known as data provenance. What is the data? Can the data be recreated from the metadata? If this criterion is satisfied then data provenance is assured. However, this is a rather difficult criterion to satisfy in practice. Consider the same ensemble 
generated on different machines, or with different codes, or even with a different algorithm. What does "the same" mean? What level of data provenance is "good enough"? The scheme should be extensible. New types of data will require new metadata and so the scheme will need to be extended. However, the scheme should be able to be modified in such a way that the new scheme includes the old scheme. Existing documents which conform to the old scheme should also conform to the new scheme.

What language should be used to construct such a scheme? Markup languages are an obvious choice. Markup languages combine text and information about that text. There are different types of markup and a particular language may combine different elements. Presentational markup is about the format of the text, for instance font type, text size or text positioning can be used to allude to relationships between different text fragments. Procedural markup contains text fragments which detail how the text is to be presented. Tex and Postscript are both procedural markup. Descriptive or semantic markup contains fragments which label other fragments but no interpretation, presentational or otherwise is mandated. HTML has both procedural and semantic elements, these pieces are more formally separated the more recent XHTML language. The semantic extensible markup language XML was chosen as it is the WC3 open standard [8] for a semantic markup language.

The metadata is contained in XML instance documents (IDs) and the rules to which these IDs must comply are described by an XML schema, called QCDml. There is a complete description of the XML standard at [8] but it is useful to consider some of the points raised in the W3-school tutorial on XML [9], which is paraphrased below.

- XML was designed to carry data, not to display data. Incompatible applications can exchange data wrapped in XML. It simplifies data transport and data sharing.

- XML is just plain text. It is easy to read and write. User defined tags allow structure to be developed. Lattice QCD data is hierarchical in nature.

- XML is designed to be self-descriptive. It can be read by a person, but see the next point!

- XML doesn't DO anything. The application decides what to do with the data. An application is required to do something with the data.

- An XML schema describes the structure of an XML ID. So an application knows how to parse an XML ID.

- XML Schemas are the successors of Document Type Definitions (DTDs). They have richer functionality, support data types and namespaces, are extensible and are themselves an XML ID.

A description of QCDml itself is given in [10] and how to markup configurations in QCDml is described in [11].

\section{QCDml now and in the future}

The biggest challenge for metadata usage is metadata capture. The moment of data creation is when all the metadata is known. Capturing this information is difficult because the goal of the 
simulation is to generate the data, not the metadata. At the time of writing, there are no codes writing QCDml as the data is generated. This is partly a consequence of XML parsers being mostly written for Java. The qdp++/chroma code [12] suite does read and write XML at run-time using the libxml2 library and could, in principle be adapted to write QCDml. However, for most codes, it would require a significant coding effort to adapt and critically maintain, a QCDml parsing interface, when the same effort could be deployed on physics functionality or code performance. Most applications write some metadata when the data is written, possibly even a simple plain text file. This then has to be converted to QCDml at a later point. Whilst this is better than not recording metadata, post-processing data is laborious and often ad-hoc, in that it doesn't happen automatically. It usually requires the intervention of an individual, when they are not too busy with something else. This means there is often a significant delay which results in an onerous task on the individual concerned ${ }^{2}$ or worse result in a loss of the metadata.

QCDml has a rich description of the action used to generate an ensemble, and some information regarding who, on which machine, with which code, but doesn't contain a lot of information regarding the algorithm. QCDml doesn't ensure data provenance, certainly, the data cannot be recreated from the metadata alone. However, the data can be discovered from the metadata by asking physics questions, so the first requirement for the metadata is satisfied even if the second isn't.

There are no plans to significantly alter QCDml, and the situation with metadata capture is also unlikely to change. However, a possible solution to these difficulties can be found by adopting some workflow tools. Besides automating the process of data generation workflow tools record all information about the stages of the calculation automatically. This helps with data provenance, and with metadata capture. This doesn't solve both problems, but provides a basis for the automation of metadata capture and increases the amount of data provenance. The reader is referred to the work of Simone's group at Fermilab [13].

Several groups have expressed interest in using the ILDG to transport or share other types of lattice data such as propagators. The ILDG working groups have adopted the USQCD propagator formats, with an extension proposed by LDG for twisted mass propagators. Metadata for this type of data has yet to be agreed, but a very lightweight scheme, i.e. one with minimal content, is desired by the interested parties. The ILDG hope to adopt such a scheme in the near future.

\section{Conclusions}

In the last decade Lattice QCD data has undergone a dramatic growth in complexity. The rise of the Mega-collaboration to cope with the increased amount of work required to complete calculations has also lead to the data being geographically distributed. Managing the data "by hand" is no longer scalable. Tools are required to automate the logistics of data management and to provide data provenance. Regional grids were developed in response to this need and the ILDG sits on top of these architectures. Remote data can be accessed with local tools, but data standards are required, which in turn enforces data discipline to curate the data. This is probably a good discipline to have. It is hoped the ILDG can encourage scientific innovation as making data readily

\footnotetext{
${ }^{2}$ The author personal experience of this.
} 
accessible so that good ideas are not thwarted by a lack of opportunity to exploit them. Many of the large collaborations are making their data available in this way, which can only be of benefit to the whole community, but this altruistic effort from both collaborations and individuals should be recognised.

\section{References}

[1] M.G. Beckett et al.,"Building the International Lattice Data Grid" [arXiv:0910.1692 [hep-lat]]

[2] http://www.usqcd.org/

[3] http://qcd.nersc.gov/

[4] http://www.globus.org/

[5] C. T. H. Davies, A. C. Irving, R. D. Kenway and C. M. Maynard [UKQCD collaboration], Nucl. Phys. Proc. Suppl. 119 (2003) 225 [arXiv:hep-lat/0209121].

[6] http://ildg.sasr.edu.au/Plone/ildg/

[7] http://ildg.sasr.edu.au/Plone/ildg/user-documentation/joining-ILDG-VO.txt/view

[8] http://www.w3.org/XML/

[9] http://www.w3schools.com/xml/default.asp

[10] C. M. Maynard and D. Pleiter, Nucl. Phys. Proc. Suppl. 140 (2005) 213 [arXiv:hep-lat/0409055].

[11] P. Coddington, B. Joo, C. M. Maynard, D. Pleiter and T. Yoshie, PoS LAT2007 (2007) 048 [arXiv:0710.0230 [hep-lat]].

[12] R. G. Edwards and B. Joo [SciDAC Collaboration and LHPC Collaboration and UKQCD Collaboration], Nucl. Phys. Proc. Suppl. 140 (2005) 832 [arXiv:hep-lat/0409003].

[13] L. Piccoli et al., FERMILAB-PUB-08-557-CD; 